\title{
A generalization of Rohn's theorem on full-rank interval matrices
}

\author{
Elena Rubei \\ Dipartimento di Matematica e Informatica "U. Dini", viale Morgagni 67/A, 50134 Firenze, Italia \\ E-mail address: elena.rubei@unifi.it
}

\begin{abstract}
A general closed interval matrix is a matrix whose entries are closed connected nonempty subsets of $\mathbb{R}$, while an interval matrix is defined to be a matrix whose entries are closed bounded nonempty intervals in $\mathbb{R}$. We say that a matrix $A$ with constant entries is contained in a general closed interval matrix $\mu$ if and only if, for every $i, j$, we have that $A_{i, j} \in \mu_{i, j}$. Rhon characterized full-rank square interval matrices, that is, square interval matrices $\mu$ such that every constant matrix contained in $\mu$ is nonsingular. In this paper we generalize this result to general closed interval matrices.
\end{abstract}

\section{Introduction}

Let $p, q \in \mathbb{N}-\{0\}$; a $p \times q$ interval matrix is a $p \times q$ matrix whose entries are closed bounded nonempty intervals in $\mathbb{R}$. We say that a $p \times q$ matrix $A$ with entries in $\mathbb{R}$ is contained in $\mu$ if and only if, for every $i, j$, we have that $A_{i, j} \in \mu_{i, j}$.

On the other side, for any field $K$, a partial matrix over $K$ is defined to be a matrix where only some of the entries are given and they are elements of $K$; a completion of a partial matrix is a specification in $K$ of the unspecified entries. We say that a submatrix of a partial matrix is specified if all its entries are specified.

There are several papers both on interval matrices and on partial matrices. On partial matrices, there is a wide literature about the problem of determining the maximal and the minimal rank of the completions of a partial matrix. We quote, for instance, the papers [1], [11] and [2]. In the last, Cohen, Johnson, Rodman and Woerdeman determined the maximal rank of the completions of a partial matrix in terms of the ranks and the sizes of its maximal specified submatrices; see also [1] for the proof. The problem of determining the minimal rank of the completions of a partial matrix

2010 Mathematical Subject Classification: 15A99, 15A03

Key words: interval matrices, rank 
seems more difficult and it has been solved only in some particular cases, see for example [10, for the case of triangular matrices.

Also interval matrices have been widely studied and in particular there are several papers studying when a $p \times q$ interval matrix $\mu$ has full rank, that is when all the matrices contained in $\mu$ have rank equal to $\min \{p, q\}$. For any $p \times q$ interval matrix $\mu=\left(\left[m_{i, j}, M_{i, j}\right]\right)_{i, j}$ with $m_{i, j} \leq M_{i, j}$, let $C^{\mu}, \Delta^{\mu}$ and $|\mu|$ be the $p \times q$ matrices such that

$$
C_{i, j}^{\mu}=\frac{m_{i, j}+M_{i, j}}{2}, \quad \Delta_{i, j}^{\mu}=\frac{M_{i, j}-m_{i, j}}{2}, \quad|\mu|_{i, j}=\max \left\{\left|m_{i, j}\right|,\left|M_{i, j}\right|\right\}
$$

for any $i, j$. The following theorem characterizes full-rank square interval matrices:

Theorem 1. (Rohn, [4]) Let $\mu=\left(\left[m_{i, j}, M_{i, j}\right]\right)_{i, j}$ be a $p \times p$ interval matrix, where $m_{i, j} \leq M_{i, j}$ for any $i, j$. Let $Y_{p}=\{-1,1\}^{p}$ and, for any $x \in Y_{p}$, denote by $T_{x}$ the diagonal matrix whose diagonal is $x$; moreover define

$$
C_{x, y}^{\mu}=C^{\mu}-T_{x} \Delta^{\mu} T_{y}
$$

Then $\mu$ is a full-rank interval matrix if and only if, for each $x, y, x^{\prime}, y^{\prime} \in Y_{p}$,

$$
\operatorname{det}\left(C_{x, y}^{\mu}\right) \operatorname{det}\left(C_{x^{\prime}, y^{\prime}}^{\mu}\right)>0
$$

See [4] and 5] for other characterizations. Finally, as to interval matrices, we want to quote also the paper [3], where an algorithm for checking regularity of interval matrices is presented, and the following theorem characterizing full-rank $p \times q$ interval matrices, see [6], [7], [9]:

Theorem 2. (Rohn) A $p \times q$ interval matrix $\mu$ with $p \geq q$ has full rank if and only if the system of inequalities

$$
\left|C^{\mu} x\right| \leq \Delta^{\mu}|x|, \quad x \in \mathbb{R}^{q}
$$

has only the trivial solution $x=0$.

Obviously the problem of partial matrices in the real case and the one of interval matrices are connected; in fact we can consider matrices whose entries are closed connected subsets of $\mathbb{R}$; these matrices generalize both the interval matrices and the partial matrices. We call a matrix whose entries are closed connected subsets of $\mathbb{R}$ a "general closed interval matrix".

In this paper we generalize Rhon's result on full-rank square interval matrices to general closed interval matrices, see Theorem 16 for the precise statement.

\section{Notation and some recalls}

- Let $\mathbb{R}_{>0}$ be the set $\{x \in \mathbb{R} \mid x>0\}$ and let $\mathbb{R}_{\geq 0}$ be the set $\{x \in \mathbb{R} \mid x \geq 0\}$; we define analogously $\mathbb{R}_{<0}$ and $\mathbb{R}_{\leq 0}$.

- Throughout the paper let $p, q \in \mathbb{N}-\{0\}$.

- Let $\Sigma_{p}$ be the set of the permutations on $\{1, \ldots, p\}$. 
- Let $M(p \times q, \mathbb{R})$ denote the set of the $p \times q$ matrices with entries in $\mathbb{R}$. For any $A \in M(p \times q, \mathbb{R})$, let $r k(A)$ denote the rank of $A$ and let $A^{(j)}$ be the $j$-th column of $A$.

- For any $p \times q$ general closed interval matrix $\mu$, any $\left\{i_{1}, \ldots, i_{s}\right\} \subset\{1, \ldots, p\}$ and any $\left\{j_{1}, \ldots, j_{r}\right\} \subset$ $\{1, \ldots, q\}$, we denote by $\mu_{i_{1}, \ldots, i_{s}, j_{1}, \ldots, j_{s}}$ the matrix obtained from $\mu$ by deleting the rows $i_{1}, \ldots, i_{s}$ and the columns $j_{1}, \ldots, j_{r}$.

Definition 3. A general interval matrix is a matrix whose entries are connected nonempty subsets of $\mathbb{R}$.

A general closed interval matrix is a matrix whose entries are closed connected nonempty subsets of $\mathbb{R}$.

An interval matrix is a matrix whose entries are closed bounded nonempty intervals of $\mathbb{R}$.

Let $\mu$ be a $p \times q$ general interval matrix. As we have already said, given a matrix $A \in M(p \times q, \mathbb{R})$, we say that $A \in \mu$ if and only if $a_{i, j} \in \mu_{i, j}$ for any $i, j$.

We define

$$
\begin{aligned}
& \operatorname{mrk}(\mu)=\min \{r k(A) \mid A \in \mu\}, \\
& \operatorname{Mrk}(\mu)=\max \{r k(A) \mid A \in \mu\} .
\end{aligned}
$$

We call them respectively minimal rank and maximal rank of $\mu$. Moreover, we define

$$
r k \operatorname{Range}(\mu)=\{r k(A) \mid A \in \mu\}
$$

we call the set above the rank range of $\mu$.

We say that the entry $i, j$ of $\mu$ is a constant if $\mu_{i, j}$ is a subset of $\mathbb{R}$ given by only one element.

We say that the entry $i, j$ of $\mu$ is bounded if $\mu_{i, j}=[a, b]$ for some $a, b \in \mathbb{R} ;$ we say that the entry $i, j$ of $\mu$ is half-bounded if either $\mu_{i, j}=[a,+\infty)$ (left-bounded) or $\mu_{i, j}=(-\infty, a]$ (right-bounded) for some $a \in \mathbb{R}$.

Remark 4. Let $\mu$ be an interval matrix. Observe that

$$
\operatorname{rkRange}(\mu)=[\operatorname{mrk}(\mu), \operatorname{Mrk}(\mu)] \cap \mathbb{N} .
$$

See [8] for a proof.

Definition 5. Given a $p \times p$ interval matrix, $\nu$, a partial generalized diagonal (pg-diagonal for short) of length $k$ of $\nu$ is a $k$-uple of the kind

$$
\left(\nu_{i_{1}, j_{1}}, \ldots, \nu_{i_{k}, j_{k}}\right)
$$

for some $\left\{i_{1}, \ldots i_{k}\right\}$ and $\left\{j_{1}, \ldots, j_{k}\right\}$ subsets of $\{1, \ldots, p\}$.

Its complementary matrix is defined to be the submatrix of $\mu$ given by the rows and columns whose indices are respectively in $\{1, \ldots, p\}-\left\{i_{1}, \ldots, i_{k}\right\}$ and in $\{1, \ldots, p\}-\left\{j_{1}, \ldots, j_{k}\right\}$.

We say that a pg-diagonal is totally nonconstant if and only if all its entries are not constant.

We define $\operatorname{det}^{c}(\mu)$ as follows:

$$
\operatorname{det}^{c}(\mu)=\sum_{\sigma \in \Sigma_{p} \text { s.t. } \mu_{1, \sigma(1)}, \ldots, \mu_{p, \sigma(p)} \text { are constant }} \epsilon(\sigma) \mu_{1, \sigma(1)} \cdots \cdot \mu_{p, \sigma(p)},
$$

where $\epsilon(\sigma)$ denotes the sign of the permutation $\sigma$. 
The following theorem and corollary were proved in [8] for interval matrices; the same proof yields the results for general closed interval matrices:

Theorem 6. Let $\mu$ be a $p \times p$ general closed interval matrix. Then $M r k(\mu)<p$ if and only if the following conditions hold:

(1) in $\mu$ there is no totally nonconstant pg-diagonal of length $p$,

(2) the complementary matrix of every totally nonconstant pg-diagonal of length between 0 and $p-1$ has det ${ }^{c}$ equal to 0.

Corollary 7. Let $\mu$ be a general closed interval matrix. Then Mrk $(\mu)$ is the maximum of the natural numbers $t$ such that there is a $t \times t$ submatrix of $\mu$ either with a totally nonconstant pgdiagonal of length $t$ or with a totally nonconstant pg-diagonal of length between 0 and $t-1$ whose complementary matrix has det $^{c} \neq 0$.

Notation 8. Let $\mu$ be a general closed interval matrix.

- We denote by $\tilde{\mu}$ the matrix obtained from $\mu$ by replacing the entries $(-\infty,+\infty)$ with 0 .

- We denote by $\bar{\mu}$ the matrix obtained from $\mu$ by replacing every entry of kind $[a,+\infty)$, for some $a \in \mathbb{R}$, with $a$ and every entry of kind $(-\infty, b]$, for some $b \in \mathbb{R}$, with $b$.

- We denote by $\mu_{l}$ the matrix obtained from $\mu$ by replacing every entry of kind $[a, b]$, for some $a, b \in \mathbb{R}$ with $a \leq b$, with $a$. We denote by $\mu_{r}$ the matrix obtained from $\mu$ by replacing every entry of kind $[a, b]$, for some $a, b \in \mathbb{R}$ with $a \leq b$, with $b$.

Definition 9. Let $\mu$ be a general closed interval matrix.

We say that $\gamma$ is a vertex matrix of $\mu$ if $\gamma_{i, j} \in\left\{m_{i, j}, M_{i, j}\right\}$ for any $i, j$ such that $\mu_{i, j}$ is a bounded interval and $\gamma_{i, j}=\mu_{i, j}$ otherwise.

We say that a vertex matrix $\gamma$ of $\mu$ is of even type if, for every $2 \times 2$ submatrix of $\mu$ such that all its entries are bounded intervals, either the number of the entries of the corresponding submatrix of $\gamma$ that are equal to the minimum of the corresponding entries of $\mu$ is even or some of its entries are constant. We say that it is of odd type if it is not of even type.

Example. Let

$$
\mu=\left(\begin{array}{ccc}
{[1,2]} & {[2,3]} & {[2,+\infty]} \\
{[-3,4]} & {[-1,5]} & {[1,4]}
\end{array}\right)
$$

Then $\gamma=\left(\begin{array}{ccc}1 & 2 & {[2,+\infty]} \\ 4 & 5 & 1\end{array}\right)$ is a vertex matrix of $\mu$ of even type, while $\delta=\left(\begin{array}{ccc}1 & 3 & {[2,-\infty)} \\ 4 & 5 & 1\end{array}\right)$ is a vertex matrix of $\mu$ of odd type.

Observe that, by using Definition 9, we can restate Rohn's theorem as follows:

Theorem 10. (Rohn, [4]) Let $\mu$ be a $p \times p$ interval matrix. Then $\mu$ is a full-rank interval matrix if and only if, for any vertex matrices $A_{1}, A_{2}$ of even type of $\mu$,

$$
\operatorname{det}\left(A_{1}\right) \operatorname{det}\left(A_{2}\right)>0
$$




\section{The main result}

Remark 11. Let $p\left(x_{1}, \ldots, x_{n}\right)$ be a polynomial with coefficients in $\mathbb{R}$ of degree 1 in every variable. Let $\bar{x} \in \mathbb{R}^{n}$. Then $p(x) \geq 0$ (respectively $p(x)>0$ ) for every $x \in \bar{x}+\mathbb{R}_{\geq 0}^{n}$ if and only if $p(\bar{x}) \geq 0$ (respectively $p(\bar{x})>0$ ) and $\frac{\partial p}{\partial x_{i}}(x) \geq 0$ for every $x \in \bar{x}+\mathbb{R}_{\geq 0}^{n}$ and for every $i=1, \ldots, n$.

Lemma 12. Let $\mu$ be a general closed interval $p \times p$ matrix. Then $\mu$ is full-rank if and only if $\tilde{\mu}$ is full-rank and for every $i, j$ such that $\mu_{i, j}=(-\infty,+\infty)$ we have that $\operatorname{Mrk}\left(\mu_{\hat{i}, \hat{j}}\right)<p-1$.

Proof. Suppose $\mu$ is full-rank. Then obviously $\tilde{\mu}$ is full-rank. Moreover, let $i, j$ be such that $\mu_{i, j}=$ $(-\infty,+\infty)$. Then the determinant of every $A \in \mu_{\hat{i}, \hat{j}}$ must be zero; otherwise, suppose there exists $A \in \mu_{\hat{i}, \hat{j}}$ with $\operatorname{det}(A) \neq 0$; then, for any choice of $x_{i, s}$ in $\mu_{i, s}$ for any $s \in\{1, \ldots, p\}-\{j\}$ and $x_{r, j}$ in $\mu_{r, j}$ for any $r \in\{1, \ldots, p\}-\{i\}$, we can choose $x_{i, j} \in \mu_{i, j}$ such that, if we define $X$ to be the matrix such that $X_{r, s}=x_{r, s}$ for $(r, s)$ such that either $r=i$ or $s=j$ and $X_{\hat{i}, \hat{j}}=A$, we have that the determinant of $X$ is zero, which is absurd since $\mu$ is full-rank. So $\operatorname{Mrk}\left(\mu_{\hat{i}, \hat{j}}\right)<p-1$ and we have proved the right-handed implication.

On the other side, let $\left(i_{1}, j_{1}\right), \ldots,\left(i_{s}, j_{s}\right)$ be the indices of the entries of $\mu$ equal to $(-\infty,+\infty)$ and suppose that $\tilde{\mu}$ is full-rank and that $\operatorname{Mrk}\left(\mu_{\hat{i_{k}}, \hat{j_{k}}}\right)<p-1$ for every $k=1, \ldots, s$. Let $B \in M(p \times p, \mathbb{R})$ be such that $B \in \mu$. For every $k=1, \ldots, s$, let $B_{k}$ be the matrix obtained from $B$ by replacing the entries $\left(i_{1}, j_{1}\right), \ldots,\left(i_{k}, j_{k}\right)$ with 0 . Then $\operatorname{det}(B)=\operatorname{det}\left(B_{1}\right)=\ldots=\operatorname{det}\left(B_{s}\right)$ since $\operatorname{Mrk}\left(\mu_{i_{k}, \hat{j_{k}}}\right)<p-1$ for every $k=1, \ldots, s$. Obviously $B_{s} \in \tilde{\mu}$, so $\operatorname{det}\left(B_{s}\right)$ is nonzero since $\tilde{\mu}$ is full-rank; hence $\operatorname{det}(B)$ is nonzero and we conclude.

Lemma 13. Let $\nu$ be a general closed interval $p \times p$ matrix with only bounded or half-bounded entries. Then $\nu$ is full-rank if and only if, for any $\gamma$ vertex matrix of $\nu$ of even type, we have that $\gamma$ is full-rank and $\operatorname{det}(\bar{\gamma})$ has the same sign as $\operatorname{det}\left(\bar{\nu}_{l}\right)$.

Proof. $\Longrightarrow$ Obviously if $\nu$ has full-rank, then the determinant of all the matrices contained in $\nu$ must have the same sign (since the determinant is a continous function and the image by a continous function of a connected subset is connected), in particular $\operatorname{det}(\bar{\gamma})$ must have the same sign as $\operatorname{det}\left(\bar{\nu}_{l}\right)$. $\Longleftarrow$ Let $A \in M(p \times p, \mathbb{R})$ be such that $A \in \nu$; define $\alpha$ to be the interval matrix such that $\alpha_{i, j}=\nu_{i, j}$ if $\nu_{i, j}$ is bounded and $\alpha_{i, j}=a_{i, j}$ if $\nu_{i, j}$ is half-bounded.

To prove that $A$ has full-rank, obviously it is sufficient to prove that $\alpha$ is full-rank. By Rohn's theorem (see Theorem [10), to prove that $\alpha$ is full-rank, it is sufficient to prove that any two vertex matrices $A_{1}, A_{2}$ of even type of $\alpha$ are full-rank and the sign of their determinant is the same. Obviously, for $i=1,2$, the matrix $A_{i}$ is contained in a vertex matrix $\gamma_{i}$ of even type of $\nu$; by assumption, for $i=1,2$, the matrix $\gamma_{i}$ is full-rank, so the matrix $A_{i}$ is full-rank and its determinant has the same sign as $\operatorname{det}\left(\bar{\gamma}_{i}\right)$. Moreover, by assumption the sign of $\operatorname{det}\left(\bar{\gamma}_{i}\right)$ is equal to the sign of $\operatorname{det}\left(\bar{\nu}_{l}\right)$ for $i=1,2$; in particular $\operatorname{det}\left(\bar{\gamma}_{1}\right)$ and $\operatorname{det}\left(\bar{\gamma}_{2}\right)$ have the same sign, so $\operatorname{det}\left(A_{1}\right)$ and $\operatorname{det}\left(A_{2}\right)$ have the same sign, as we wanted to prove.

Notation 14. Let $\rho$ be a square general closed interval matrix. We say that DET $(\rho)$ is greater than 0 (respectively less than 0 , equal to $0 \ldots$ ) if and only if, for any $A \in \rho$, we have that $\operatorname{det}(A)$ is greater than 0 (respectively less than 0 , equal to $0 \ldots$ ). 
Lemma 15. Let $\rho$ be a general closed interval $p \times p$ matrix with only constant or half-bounded entries. Then $\rho$ is full-rank if and only if $\operatorname{det}(\bar{\rho}) \neq 0$ and, for any $\left(i_{1}, j_{1}\right), \ldots,\left(i_{s}, j_{s}\right) \in\{1, \ldots, p\} \times\{1, \ldots, p\}$ such that $i_{1}, \ldots, i_{s}$ are distinct and $j_{1}, \ldots, j_{s}$ are distinct and $\rho_{i_{1}, j_{1}}, \ldots, \rho_{i_{s}, j_{s}}$ are half-bounded,

$$
(-1)^{i_{1}+j_{1}+\tilde{i_{2}}+\tilde{j_{2}}+\ldots+\widetilde{i_{s}}+\widetilde{j_{s}}+\chi\left(\left(i_{1}, j_{1}\right), \ldots,\left(i_{s}, j_{s}\right)\right)} \operatorname{det}(\bar{\rho}) \operatorname{det}\left(\bar{\rho}_{i_{1}, \ldots, i_{s},} \widehat{j_{1}, \ldots, j_{s}}\right) \geq 0
$$

where:

- the determinant of a $0 \times 0$ matrix is defined to be 1 ,

- $\chi\left(\left(i_{1}, j_{1}\right), \ldots,\left(i_{s}, j_{s}\right)\right)$ is defined to be the number of the right-bounded intervals in $\rho_{i_{1}, j_{1}}, \ldots, \rho_{i_{s}, j_{s}}$,

- for $t=2, \ldots, s$, we define $\widetilde{i_{t}}$ to be $i_{t}$ minus the number of the elements among $i_{1}, \ldots i_{t-1}$ smaller than $i_{t}$ and $\widetilde{j}_{t}$ to be $j_{t}$ minus the number of the elements among $j_{1}, \ldots j_{t-1}$ smaller than $j_{t}$.

Proof. By Remark 11, the matrix $\rho$ is full-rank if and only if $\operatorname{det}(\bar{\rho}) \neq 0$ and, for any $i_{1}, j_{1}$ such that $\rho_{i_{1}, j_{1}}$ is half-bounded,

$$
(-1)^{i_{1}+j_{1}+\chi\left(i_{1}, j_{1}\right)} \operatorname{det}(\bar{\rho}) \operatorname{DET}\left(\rho_{\hat{i_{1}}, \hat{j_{1}}}\right) \geq 0
$$

and, again by Remark 11, the last condition holds if and only if, for any $i_{1}, j_{1}$ such that $\rho_{i_{1}, j_{1}}$ is half-bounded, we have that

$$
(-1)^{i_{1}+j_{1}+\chi\left(i_{1}, j_{1}\right)} \operatorname{det}(\bar{\rho}) \operatorname{det}\left(\bar{\rho}_{\hat{i}_{1}, \hat{j}_{1}}\right) \geq 0
$$

and, for any $i_{2}, j_{2}$ such that $i_{1} \neq i_{2}$ and $j_{1} \neq j_{2}$ and $\rho_{i_{2}, j_{2}}$ is half-bounded, we have that

$$
(-1)^{i_{1}+j_{1}+\tilde{i_{2}}+\tilde{j_{2}}+\chi\left(\left(i_{1}, j_{1}\right),\left(i_{2}, j_{2}\right)\right)} \operatorname{det}(\bar{\rho}) \operatorname{DET}\left(\rho_{\widehat{i_{1}, i_{2}}, \widehat{j_{1}, j_{2}}}\right) \geq 0
$$

and so on.

Theorem 16. Let $\mu$ be a general closed interval matrix. Then $\mu$ is full-rank if and only if the following conditions hold:

(1) for every $i, j$ such that $\mu_{i, j}=(-\infty,+\infty)$ we have that in $\mu_{\hat{i}, \hat{j}}$ there are no totally nonconstant pg-diagonal of length $p-1$ and the complementary matrix of every totally nonconstant pg-diagonal in $\mu_{\hat{i}, \hat{j}}$ of length between 0 and $p-2$ has det $^{c}$ equal to 0.

(2) the product of the determinant every vertex matrix of $\overline{\tilde{\mu}}$ of even type and the determinant of $\overline{\tilde{\mu}}_{l}$ is positive,

(3) for every vertex matrix $\gamma$ of $\tilde{\mu}$ of even type and every $\left(i_{1}, j_{1}\right), \ldots,\left(i_{s}, j_{s}\right) \in\{1, \ldots, p\} \times\{1, \ldots, p\}$ such that $i_{1}, \ldots, i_{s}$ are distinct and $j_{1}, \ldots, j_{s}$ are distinct and $\mu_{i_{1}, j_{1}}, \ldots, \mu_{i_{s}, j_{s}}$ are half-bounded, we have:

$$
(-1)^{i_{1}+j_{1}+\widetilde{i_{2}}+\widetilde{j_{2}}+\ldots+\widetilde{i_{s}}+\widetilde{j_{s}}+\chi\left(\left(i_{1}, j_{1}\right), \ldots,\left(i_{s}, j_{s}\right)\right)} \operatorname{det}\left(\overline{\tilde{\mu}}_{l}\right) \operatorname{det}\left(\bar{\gamma}_{i_{1}, \ldots, i_{s},}, \widehat{j_{1}, \ldots, j_{s}}\right) \geq 0
$$

where:

- the determinant of a $0 \times 0$ matrix is defined to be 1 ,

- the number $\chi\left(\left(i_{1}, j_{1}\right), \ldots,\left(i_{s}, j_{s}\right)\right)$ is defined to be the number of right-bounded intervals in $\mu_{i_{1}, j_{1}}, \ldots, \mu_{i_{s}, j_{s}}$,

- for $t=2, \ldots, s$, we define

$$
\begin{gathered}
\widetilde{i_{t}}:=i_{t}-\sharp\left\{i_{r} \text { for } r=1, \ldots, t-1 \mid i_{r}<i_{t}\right\} \\
\widetilde{j_{t}}:=j_{t}-\sharp\left\{j_{r} \text { for } r=1, \ldots, t-1 \mid j_{r}<j_{t}\right\} .
\end{gathered}
$$


Proof. By Lemma 12, the matrix $\mu$ is full-rank if and only if $\tilde{\mu}$ is full-rank and for every $i, j$ such that $\mu_{i, j}=(-\infty,+\infty)$ we have that $\operatorname{Mrk}\left(\mu_{\hat{i}, \hat{j}}\right)<p-1$. By Theorem 6 this is true if and only if $\tilde{\mu}$ is full-rank and for every $i, j$ such that $\mu_{i, j}=(-\infty,+\infty)$ we have that in $\mu_{\hat{i}, \hat{j}}$ there are no totally nonconstant pg-diagonal of length $p-1$ and the complementary matrix of every totally nonconstant pg-diagonal in $\mu_{\hat{i}, \hat{j}}$ of length between 0 and $p-2$ has $\operatorname{det}^{c}$ equal to 0 .

Moreover, by Lemma 13 (with $\nu=\tilde{\mu}$ ), we have that $\tilde{\mu}$ is full-rank if and only if, for any $\gamma$ vertex matrix of $\tilde{\mu}$ of even type, we have that $\gamma$ is full-rank and $\operatorname{det}(\bar{\gamma})$ has the same sign as $\operatorname{det}\left(\overline{\tilde{\mu}}_{l}\right)$.

Finally, this is true if and only if (2) holds and, by Lemma 15, for any $\gamma$ vertex matrix of $\tilde{\mu}$, for any $\left(i_{1}, j_{1}\right), \ldots,\left(i_{s}, j_{s}\right) \in\{1, \ldots, p\} \times\{1, \ldots, p\}$ such that $i_{1}, \ldots, i_{s}$ are distinct and $j_{1}, \ldots, j_{s}$ are distinct and $\gamma_{i_{1}, j_{1}}, \ldots, \gamma_{i_{s}, j_{s}}$ are half-bounded, we have that $\operatorname{det}(\bar{\gamma}) \neq 0$ and

$$
(-1)^{i_{1}+j_{1}+\tilde{i_{2}}+\tilde{j_{2} \ldots}+\tilde{i_{s}}+\tilde{j_{s}}+\chi\left(\left(i_{1}, j_{1}\right), \ldots,\left(i_{s}, j_{s}\right)\right)} \operatorname{det}(\bar{\gamma}) \operatorname{det}\left(\bar{\gamma}_{i_{1}, \ldots, i_{s}, j_{1}, \ldots, j_{s}}\right) \geq 0
$$

which, by condition (2), is equivalent to condition (3).

Examples. 1) Let

$$
\alpha=\left(\begin{array}{ccccc}
(-\infty,+\infty) & {[1,+\infty)} & 1 & 1 & 4 \\
1 & {[2,3]} & 6 & 2 & 4 \\
(-\infty, 2] & 0 & {[1,4]} & 0 & {[3,6]} \\
0 & {[-1,2]} & 3 & 1 & 2 \\
3 & 0 & 3 & 1 & 2
\end{array}\right)
$$

We can easily that $\alpha$ does not satisfy condition (2) of Theorem [16, in fact $\overline{\tilde{\alpha}}_{l}=\left(\begin{array}{ccccc}0 & 1 & 1 & 1 & 4 \\ 1 & 2 & 6 & 2 & 4 \\ 2 & 0 & 1 & 0 & 3 \\ 0 & -1 & 3 & 1 & 2 \\ 3 & 0 & 3 & 1 & 2\end{array}\right)$ has negative determinant, while the following vertex matrix of even type of $\overline{\tilde{\alpha}}$ has positive determinant:

$$
\left(\begin{array}{lllll}
0 & 1 & 1 & 1 & 4 \\
1 & 2 & 6 & 2 & 4 \\
2 & 0 & 1 & 0 & 3 \\
0 & 2 & 3 & 1 & 2 \\
3 & 0 & 3 & 1 & 2
\end{array}\right)
$$

So $\alpha$ is not full-rank. In fact it contains the matrix

$$
\left(\begin{array}{ccccc}
0 & 1 & 1 & 1 & 4 \\
1 & 2 & 6 & 2 & 4 \\
2 & 0 & 1 & 0 & 3 \\
0 & 6 / 5 & 3 & 1 & 2 \\
3 & 0 & 3 & 1 & 2
\end{array}\right)
$$

which is not invertible. 
2) Let

$$
\beta=\left(\begin{array}{cccc}
{[2,+\infty)} & 1 & 2 & (-\infty,+\infty) \\
{[1,2]} & 0 & 3 & 2 \\
3 & {[3,7]} & 5 & 3 \\
0 & 0 & 0 & {[1,+\infty)}
\end{array}\right)
$$

We can easily see that $\beta$ satisfies conditions $(1),(2),(3)$ of Theorem 16, so it is full-rank. 3) Let

$$
\delta=\left(\begin{array}{cccc}
(-\infty,+\infty) & 1 & 2 & (-\infty,+\infty) \\
{[1,2]} & {[1,2]} & 9 & 2 \\
3 & {[1,5]} & 4 & 0 \\
2 & {[1,2]} & {[-1,+\infty]} & 3
\end{array}\right)
$$

Obviously it does not satisfy condition (1) of Theorem 16, in fact $\delta_{\hat{1}, \hat{1}}$ contains totally nonconstant pg-diagonal whose complementary matrix has $d e t^{c} \neq 0$. So $\delta$ is not full-rank.

Open problem. A problem that naturally arises is the one of the characterization of full-rank matrices whose entries are (not necessarily closed) connected subsets of $\mathbb{R}$.

Acknowledgments. This work was supported by the National Group for Algebraic and Geometric Structures, and their Applications (GNSAGA-INdAM).

\section{References}

[1] Cohen, N.; Dancis, J. Maximal Ranks Hermitian Completions of Partially specified Hermitian matrices. Linear Algebra Appl. 244 (1996), 265-276.

[2] Cohen, N.; Johnson, C.R.; Rodman, Leiba; Woerdeman, H. J. Ranks of completions of partial matrices. The Gohberg anniversary collection, Vol. I (Calgary, AB, 1988), 165-185, Oper. Theory Adv. Appl., 40, Birkhäuser, Basel, 1989.

[3] Jansson, C.; Rohn, J. An algorithm for checking regularity of interval matrices, SIAM J. Matrix Anal. Appl. 20 (1999), no. 3, 756-776.

[4] Rohn, J. Systems of Linear Interval Equations. Linear Algebra Appl. 126 (1989) 39-78.

[5] Rohn, J. Forty necessary and sufficient conditins for regularity of interval matrices: A survey. Electronic Journal of Linear Algebra 18 (2009).

[6] Rohn, J. A Handbook of Results on Interval Linear Problems, Prague: Insitute of Computer Science, Academy of Sciences of the Czech Republic, 2012.

[7] Rohn, J. Enclosing solutions of overdetermined systems of linear interval equations, Reliable Computing, 2 (1996), 167-171.

[8] Rubei, E. On rank range of interval matrices, arXiv:1712.09940 
[9] Shary, S.P. On Full-Rank Interval Matrices Numerical Analysis and Applications 7 (2014), no. 3, 241-254.

[10] Woerdeman, H. J. The lower order of lower triangular operators and minimal rank extensions. Integral Equations and Operator Theory 10 (1987), 859-879.

[11] Woerdeman, H. J. Minimal rank completions for block matrices. Linear algebra and applications (Valencia, 1987). Linear Algebra Appl. 121 (1989), 105-122. 